\title{
Monte Carlo Simulation of Grafting Reactions of Styrene onto Cellulose Acetate
}

\author{
Tamio Yasukawa, Yasunori Sasaki, and Kenkichi Murakami \\ Chemical Research Institute of Non-Aqueous Solutions, \\ Tohoku University, Sendai, Japan. \\ Katsuyuki Ogura and Hiroshi Sobue \\ Department of Industrial Chemistry, Faculty of Engineering, \\ Seikel University, Musashino, Tokyo, Japan.
}

(Received January 23, 1973)

\begin{abstract}
KEY WORDS Monte Carlo Calculation / Simulation / Grafting Reaction / Styrene / Cellulose Acetate / Molecular Weight Distribution /
\end{abstract}

In a previous paper, it was suggested that the molecular weight distribution of graft chains could be estimated from simulation of grafting reactions. ${ }^{1}$ Some results of simulations for the polyethylene-styrene system have been published. ${ }^{2}$ In the present work, we attempted to investigate which of several models is most acceptable for the simulation of grafting reactions in the cellulose acetate-styrene system, where the measurement of the molecular weight distribution of graft chains is feasible.

Cellulose acetate films irradiated with gamma rays at a dose rate of $2 \times 10^{5} \mathrm{r} / \mathrm{hr}$ for $20 \mathrm{hr}$ in air were reached with styrene in methanol $(1: 1$ by volume) at $50^{\circ} \mathrm{C}$ under nitrogen. Details of grafting procedures are described elsewhere. ${ }^{3}$

Kinetic data on the grafting reaction were obtained by a method similar to the one employed for the polyethylene-styrene system. ${ }^{1}$ In Figure 1 are shown the growth rate of the graft chain (abbreviated $G R$ ), the probability of deactivation of graft radicals $(P R O B)$, and the differential amounts of trunk polymer radicals which participate in the grafting reaction $(D A C T)$ for the present system.

In the polymerization of styrene, transfer to monomer and disproportionation are insignificant. ${ }^{4}$ Transfer to methanol and cellulose acetate will also be negligible. Hence, we

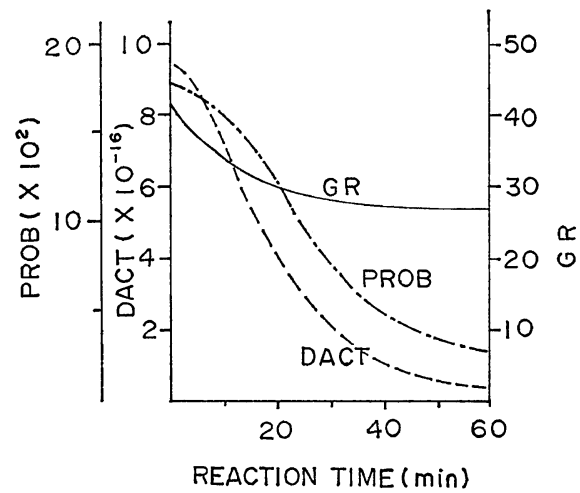

Figure 1. Kinetic data for grafting of styrene onto cellulose acetate at $50^{\circ} \mathrm{C}: G R$, growth rate $\left(\mathrm{min}^{-1}\right) ; P R O B$, probability of deactivation $\left(\mathrm{min}^{-1}\right)$; $D A C T$, differential amounts of active sites $\left(\mathrm{min}^{-1}\right.$, per gram of cellulose acetate).

tentatively assumed that the polystyryl graft radicals are usually terminated by coupling. The probability of coupling of polymer radicals is not always independent of chain length: e.g., the probability of coupling of the polystyryl radical in bulk polymerization at $30^{\circ} \mathrm{C}$ is approximately proportional to the inverse square root of the chain length. ${ }^{5}$ In grafting reactions, the probability of coupling may be influenced differently by chain length. Therefore five models were chosen for simulation of grafting 
reactions: the probability of coupling of active chains of length $L$ at time $t$ is expressed

$$
\begin{aligned}
& p(L, t)=C(t) \cdot F(L) \\
& \quad\left(F(L)=L, L^{-1 / 2}, L^{0}, L^{-1 / 2}, \text { or } L^{-1}\right)
\end{aligned}
$$

where $C(t)$ is a normalization constant given by the relation

$$
\sum_{L} p(L, t) \cdot n(L, t)=P R O B(t) \cdot \sum_{L} n(L, t)
$$

with $n(L, t)$ the number of active chains of length $L$ at time $t$. The growth rate was assumed to be independent of chain length in all the models.

The simulation program consists of three main blocks:

(1) the distribution of active chain lengths at time $t$ is calculated by the following relations

$$
\begin{aligned}
n(L, t)= & n(L-G R(t-1), t-1) \\
& \times(1.0-p(L-G R(t-1), t-1)) \\
n(0, t)= & D A C T(t) ;
\end{aligned}
$$

(2) a modified population of active chains is generated where the number of active chains of length $L$ is given by $p(L, t) \cdot n(L, t)$; in order to estimate the molecular weight distribution of coupled chains formed at time $t, 500$ pairs of chains are drawn from this population by the Monte Carlo technique; procedures (1) and (2) are repeated each minute until the end of reaction; and lastly (3) the molecular weight distribution of graft chains is corrected for the effects of the termination reaction by which graft radicals active at the end of reaction are terminated.

Figure 2A shows differential weight distributions obtained with the respective models, together with the distribution of isolated graft chains determined by gel permeation chromatography. Among the five models that with $L^{-1 / 2}$ agrees best with the experimental results, though the discrepancy in the high molecular weight region $\left(>2 \times 10^{5}\right)$ is significant.

Hitherto, all the active sites were assumed to have the same growth rate at a given time. This assumption, however, would not be appropriate for heterogeneous reactions; e.g., some active sites such as those located on the surface of films may grow more rapidly than others, resulting in dispersion of the molecular weight

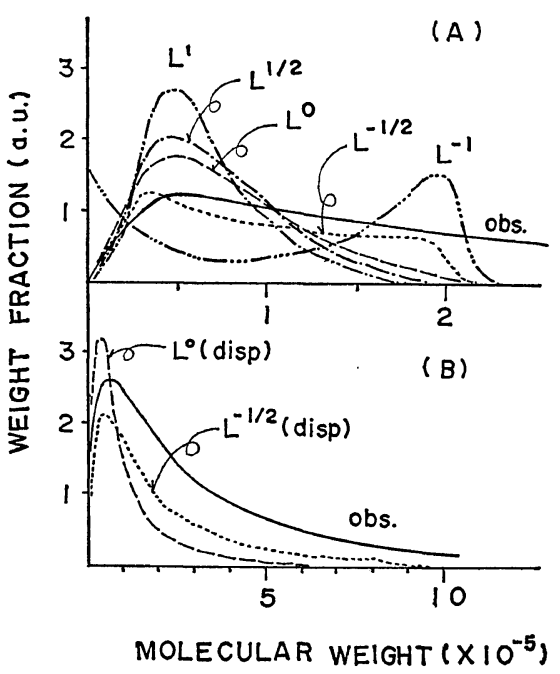

Figure 2. Differential molecular weight distribution (ordinates in arbitrary units) for polystyrene grafted at $50^{\circ} \mathrm{C}$ for 60 minutes: obs., the distribution obtained by GPC; $L^{1}-L^{-1}$, distributions obtained by simulation with models designated; (A), no dispersion in growth rates; (B), with dispersion in growth rates.

distribution. In order to investigate the effects on the molecular weight distribution of the dispersion in growth rates, several types of growth rate spectra were assumed tentatively and the molecular weight distributions were calculated. Figure 2B shows the distributions obtained with the $L^{0}$ and $L^{-1 / 2}$ models where growth rates are assumed to be distributed as follows: $50 \%$ of DACT has a growth rate $0.5 . \mathrm{GR} ; 30 \%$, GR; 7.5\%, 2.GR; 5\%, 3.GR; and $2.5 \%, 4$. GR throughout the reaction. The distribution obtained with the $L^{-1 / 2}$ (dispersion) agrees fairly well with the observation, while the $L^{0}$ (dispersion), even with several other types of dispersions in growth rates, is unsuccessful in giving acceptable results.

We may consider that the probability of coupling of polystyryl graft radicals in the present system is approximately proportional to the inverse square root of the chain length and that the growth rates are fairly disperse throughout the reaction. The rate-determining step in the coupling reaction of homopolymer radicals is considered to be the segmental motion of the active end around the center of gravity of 
the radical, rather than the translational diffusion of the chain center. ${ }^{6,7}$ Hence, it would be reasonable that the probability of coupling of graft radicals is influenced by the chain length in ways somewhat similar to homopolymer radicals.

It is noteworthy that the $L^{-1}$ model gave a distribution similar to that reported by Wellons, et $a l .,{ }^{8}$ who conducted the grafting reaction at room temperature in the presence of pyridine. The segmental motions of graft chains would be noticeably influenced by the nature of the media. Accordingly, the probability of coupling of graft radicals may be sensitive to reaction temperature and solvents.

\section{REFERENCES}

1. T. Yasukawa, T. Takahashi, K. Murakami, K.
Araki, T. Sasuga, and H. Ohmichi, J. Polymer Sci., Part A-1 10, 259 (1972).

2. T. Yasukawa, T. Takahashi, K. Murakami, K. Araki, T. Sasuga, and H. Ohmichi, Repts. Prog. Polymer Phys. Jap., 15, 507 (1972).

3. H. Sobue, K. Matsuzaki, and H. Komagata, J. Polymer Sci., Part C, 2, 415 (1963).

4. M.S. Matheson, E. E. Auer, E. B. Bevilacqua, and E. J. Hart, J. Amer. Chem. Soc., 73, 1700 (1951).

5. T. Yasukawa, T. Takahashi, and K. Murakami, J. Chem. Phys., 57, 2591 (1972).

6. S. W. Benson and A. M. North, J. Amer. Chem. Soc., 84, 935 (1962).

7. A. M. North and G. A. Reed, J. Polymer Sci., Part A-1, 1, 1311 (1963).

8. J. D. Wellons, A. Schindler, and V. Stannett, Polymer, 5, 499 (1964). 